\title{
The LHCb Turbo stream
}

\author{
A. Puig a, ${ }^{\mathrm{a},}$, on behalf of the LHCb Trigger Group \\ ${ }^{a}$ École Polytechnique Fédérale de Lausanne, Lausanne, Switzerland
}

\begin{abstract}
The LHCb experiment will record an unprecedented dataset of beauty and charm hadron decays during Run II of the LHC, set to take place between 2015 and 2018. A key computing challenge is to store and process this data, which limits the maximum output rate of the LHCb trigger. So far, $\mathrm{LHCb}$ has written out a few $\mathrm{kHz}$ of events containing the full raw sub-detector data, which are passed through a full offline event reconstruction before being considered for physics analysis. Charm physics in particular is limited by trigger output rate constraints. A new streaming strategy includes the possibility to perform the physics analysis with candidates reconstructed in the trigger, thus bypassing the offline reconstruction. In the Turbo stream the trigger will write out a compact summary of physics objects containing all information necessary for analyses. This will allow an increased output rate and thus higher average efficiencies and smaller selection biases. This idea will be commissioned and developed during 2015 with a selection of physics analyses. It is anticipated that the turbo stream will be adopted by an increasing number of analyses during the remainder of LHC Run II (2015-2018) and ultimately in Run III (starting in 2020) with the upgraded $\mathrm{LHCb}$ detector.
\end{abstract}

Keywords: LHC Run II; LHCb; Trigger; Data processing

PACS: 07.05.Fb; 13.75.Cs; 07.05; Hd; 07.05.Kf

\section{Introduction}

The LHCb detector [1, 2] is a single-arm forward spectrometer covering the pseudorapidity range $2<\eta<$ 5 , designed for the study of particles containing $b$ or $c$ quarks. The detector includes a high-precision tracking system consisting of a silicon-strip vertex detector surrounding the $p p$ interaction region, the VELO, a largearea silicon-strip detector located upstream of a dipole magnet with a bending power of about $4 \mathrm{Tm}$, and three stations of silicon-strip detectors and straw drift tubes placed downstream of the magnet. Different types of charged hadrons are distinguished using information from two ring-imaging Cherenkov detectors. Photons, electrons and hadrons are identified by a calorimeter system consisting of scintillating-pad and preshower detectors, an electromagnetic calorimeter and a hadronic calorimeter. Muons are identified by a system composed of alternating layers of iron and multiwire proportional chambers.

The online event selection is performed by a trigger [3] consisting of a hardware stage, the L0, which

\footnotetext{
${ }^{*}$ Corresponding author

Email address: albert.puig@cern.ch (A. Puig)
}

reduces the event rate to $1 \mathrm{MHz}$ from the input collision rate of at most $30 \mathrm{MHz}$ based on information from the calorimeter and muon systems, followed by a software stage, the HLT, that runs in the LHCb Event Filter Farm (EFF) and gave a final rate of $5 \mathrm{kHz}$ in Run I. This software trigger is divided into two steps, HLT1 and HLT2, that perform a partial and full event reconstruction, respectively. On average the time spent in stable running for the LHC during 2012 was around $30 \%$, due to planned technical stops and machine development phases, the ramping of the LHC dipole magnets between data taking fills, and unplanned maintenance. To increase CPU efficiency, in 2012 each EFF node was equipped with $1 \mathrm{~TB}$ of local storage space, and during data taking $20 \%$ of the L0 output was buffered in order to run the software stage during LHC downtime. Thanks to this increase in available computing power, some reconstruction thresholds could be loosened, resulting in improved reconstruction efficiency of signal candidates. The full trigger scheme used in 2012 is shown in Fig. 1.

\section{Data taking in Run II}

Due to the success of deferring the HLT processing, the deferral paradigm was expanded by making 

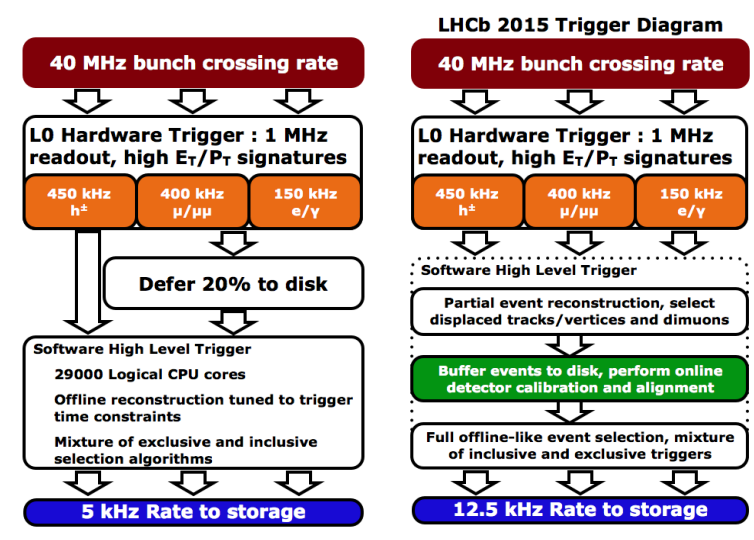

Figure 1: LHCb trigger scheme in Run I (left) including deferral and in Run II (right).

its two steps into separate processes and performing the buffering after the first level, as shown in Fig. 1. rather than buffering $20 \%$ of the L0 output [4]. To cope with the increased needs in CPU power, the EFF has been upgraded to increase the size of the local disks (the buffer) to 5 PB in total, and to double the CPU power. With the increased time allowed in the second trigger level, the reconstruction in the trigger can be brought closer to that performed offline. Additionally, automated alignment and calibration tasks are performed using the buffered data to provide observables of the same quality as achieved offline [5]; these include the alignment of the VELO and tracking stations, the RICH mirror alignment and photon detector refractive index calibration, and the global time alignment of the straw drift tubes.

\section{The Turbo stream}

Given that these calibration and alignment procedures provide offline-quality information in the trigger, it is natural to ask if an additional offline reconstruction is required, as it was done in Run I. The aim of the Turbo stream is to provide a framework in which physics analyses can be performed using the trigger reconstruction directly. For data sent to the Turbo stream, only the candidates identified by the trigger reconstruction and selection are persistified, discarding the rest of the event. This results in an event size an order magnitude smaller than that of the full event, so for Run II data taking $20 \%$ of the trigger selections will be run at a cost of less than $2 \%$ of the total bandwidth.

In Run I, some limited information from physics objects built in the trigger was already stored in the raw event through HLT object summaries. In the Turbo stream, these summaries have been expanded to store full, selected decay chains and any $\mathrm{C}++$ physics object class necessary for data analysis as key-value pairs. This new method gives great flexibility, since the persisted data can be chosen to save memory and avoid duplication.

In Run II, expanded object summaries are built (and persisted to disk) for those candidates selected in the Turbo stream; at the analysis stage, the same converter tool is used to resurrect the saved objects and rebuild the physics objects selected by the trigger. The list of keyvalue pairs used for each event can be retrieved from the event header, making it possible to process events with different lists simultaneously; this allows to preserve backwards compatibility.

\section{Conclusions and future plans}

The Turbo stream provides a novel way of storing and resurrecting candidates selected by the trigger, allowing to skip the offline reconstruction and to perform physics analysis directly using trigger quantities. This is possible thanks to the new trigger scheme introduced in Run II, which will allow to perform automatic online calibration and alignment procedures on buffered data. Approximately $20 \%$ of the trigger rate will be dedicated to this Turbo stream in Run II, but thanks to its small event size it will only represent $2 \%$ of the total bandwidth.

It is foreseen that more analyses will choose to move to the Turbo stream format as confidence is gained during the comissioning of the system. In the LHCb upgrade, an output bandwidth from the HLT to the storage of $5 \mathrm{~GB} / \mathrm{s}$ is projected, so if all events were sent to the Turbo stream, an event rate of $1 \mathrm{MHz}$ could be saved. While this is not possible-some analyses will always need the complete event and not specific candidates-a mixed solution is envisaged, with most of the rate dedicated to the Turbo stream and specific trigger selections being saved in full event format.

\section{References}

[1] A. A. Alves Jr., et al. (LHCb collaboration), The LHCb detector at the LHC, JINST 3 (2008) S08005. doi 10.1088/1748-0221/ 3/08/S08005

[2] R. Aaij, et al. (LHCb collaboration), LHCb detector performance, Int. J. Mod. Phys. A30 (2015) 1530022. doi 10.1142/ S0217751X15300227 arXiv: 1412.6352

[3] R. Aaij, et al., The LHCb trigger and its performance in 2011, JINST 8 (2013) P04022. doi 10.1088/1748-0221/8/ 04/P04022 arXiv: 1211.3055

[4] A. Dziurda, The LHCb trigger system and its upgrade, this issue (2015).

[5] Z. Xu, M. Tobin, Novel real-time alignment and calibration of the LHCb Detector in Run2, this issue (2015). 